\title{
Application of Enzyme Immobilization in the Food Industry
}

\author{
M. B. Mandake, Urvashi Das, Laxman Phad, Sakshi Busamwar* \\ Department of Chemical Engineering \\ Bharatividyapeeth College of Engineering, Kharghar, University of Mumbai, Kharghar, Navi Mumbai \\ *Corresponding Author: Sakshi Busamwar, Department of Chemical Engineering, Bharatividyapeeth \\ College of Engineering, Kharghar, University of Mumbai, Kharghar, Navi Mumbai
}

\begin{abstract}
Enzymes are complex globular proteins present in living cells where they act as catalysts that facilitate chemical changes in substances. Enzymes promote biochemical reactions in living systems. Without enzymes there can be no life. Although enzymes are only formed in living cells, many can be extracted or separated from the cells and can continue in vitro. Industrial applications of enzymes include food (baking, dairy products, starch conversion) and beverage processing (beer, wine, fruit and vegetable juices), animal feed, textiles, pulp and paper, detergents, biosensors, cosmetics, health care and nutrition, wastewater treatment, pharmaceuticals and chemical manufacture and, more recently, biofuels such as biodiesel and bioethanol. Enzymes have several benefits over classical catalyst like high selectivity, enhances the product, high efficiency, non-toxic, cost effective, etc. However, all these desirable characteristics of enzymes and their widespread industrial applications are often hampered by their lack of long-term operational stability, shelfstorage life and by their cumbersome recovery and reuse. These drawbacks can generally be overcome by immobilization of enzymes.
\end{abstract}

Keywords: Enzymes, enzyme immobilization, applications

\section{ENZYME IMMOBILIZATION}

"Immobilized enzymes" refer to an enzyme that has been confined or localized so that it can be reused continuously. The immobilized enzyme can be a free enzyme, cell or an organelle. There are many advantages of immobilized enzyme over simply enzyme like ability to be confined to a place, predetermined space, etc. in this immobilized form, the enzyme can be repeatedly and continuously used.

\subsection{Carrier Materials for Enzyme Immobilization}

The interaction between the enzyme and carrier provides an immobilized enzyme with specific chemical, biochemical, mechanical and kinetic properties. Carriers can be classified according to their morphology or their chemical composition. The support should have the properties such as large surface area and high permeability; sufficient functional groups for enzyme attachment under nondenaturing conditions; hydrophilic character; water insolubility; chemical and thermal stability; mechanical strength; high rigidity and suitable particle form; resistance to microbial attack; regenerability; toxicological safety; and low or justifiable price. Some of the examples of support material are biopolymer, inorganic polymer.

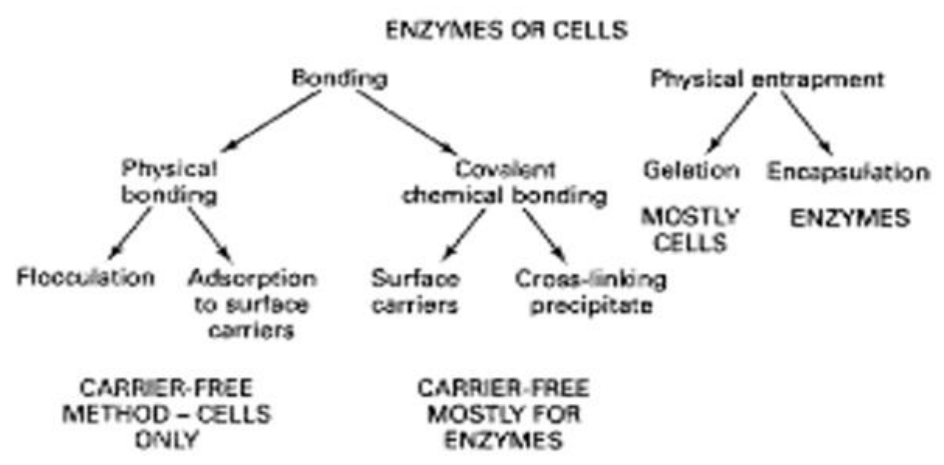

Fig1. Classification of methods of Enzyme Immobilization 


\section{METHODS OF ENZYME IMMOBILIZATION}

Enzyme or cell can be immobilization can be done in various methods.

Mainly classified as i) Bonding ii) Physical entrapped. The method to be adopted greatly influences the property of result product. Selection of method of immobilization depends upon the process specifications for the catalyst including such parameters like overall catalytic activity, effectiveness of the catalyst utilization, deactivation, regeneration characteristic and cost.

\subsection{Bonding Method}

Bonding methods are further classified as:-i) Physical Bonding. ii) Co-valent Chemical Bonding. These are very simple methods of enzyme immobilization. Typical supports for emzyme immobilization are polysaccharides, synthetic resins, biopolymers or inorganic solids such as silica or zeolites.

\subsubsection{Physical Bonding}

Physical Bonding is mainly used for cell only. It is carrier free. Flocculation and Adsorption to surface careless are the major types of Physical Bonding.

\subsubsection{Co-valent Chemical Bondings}

In covalent chemical bonging, the enzyme immobilization method is strong bond formation that occurs between the functional group on the enzyme and carrier molecules. This is mostly used for enzyme molecule may be blocked during covalent bonding.

Two types i) Surface carriers ii) Cross-linking precipitate.

\subsection{Physical Entrapment}

In this method entrapment, enzyme inclusion occurs within a mesh network. It retains the enzymes but substrate and products are allowed to pass through. There are two types of physical entrapment are i) Gelation. ii) Encapsulation.

\section{AdVANTAGE OF IMMOBILIZED ENZYMES}

- Permit the re-use of the component enzyme(s).

- Ideal for continuous operation.

- Product is enzyme free.

- Permit more accurate control of catalytic processes.

- Improve stability of enzymes.

- Allow development of a multienzyme reaction system.

- Offer considerable potential in industrial and medical use.

- Reduce effluent disposal problems.

\section{ENZYMES COMMONLY USED IN INDUSTRY}

LIPASE- Lipases (triacylglycerol acylhydrolase) act on carboxylic ester bonds, and require no cofactor. Long-chain fatty acids are the natural substrates for lipase. Lipases are of interest for industry because of their natural function of hydrolyzing triglycerides into diglycerides, monoglycerides, fatty acids, and glycerol.

AMYLASE- Enzymes which are capable of hydrolyzing the $\alpha$-1,4-glucosidic linkages of starch are called amylases (Vihinen\&Mantsiila, 1989). Although amylases are found in plants and animals, microbial amylases are most common in industry. $\alpha$-Amylase (EC 3.2.1.1) and glucoamylase (EC 3.2.1.3) are two major amylases.

PECTIC ENZYMES- These enzymes can hydrolyze the long and complicated molecules named pectins that are structural polysaccharides in the plant cell and maintain integrity of the cell wall. . Pectic substances are high molecular weight (30,000-300,000 Da), negatively charged complex polysaccharides, with a backbone of galacturonic acid residues linked by $\alpha-1,4$ - linkages (Kashyap et al., 2001). The American Chemical Society has categorized pectic substances into four main groups. The first group, called protopectins, are water-soluble pectic substances composed of pectin or pectic acid. The second group is pectic acid and polymers of galacturonans that contain negligible amounts of methoxyl groups. Pectinic acid, the third group, is the polygalacturonan chain with various amounts 
of methoxyl groups $(0-75 \%)$. Pectin is the last group and defines the mixture of differing compositions of galacturonate units esterified with methanol.

LACTASE- The enzyme $\beta$-galactosidase is also known as lactase. It is obtained from microorganisms, plants, and animals. It is used for the hydrolysis of the disaccharide sugar lactose present in milk and whey.

PROTEASES- Proteases are protein-degrading enzymes and catalyze the cleavage of peptide bonds in the proteins. Proteases are classified according to their catalytic action into endopeptidases and exopeptidases. Proteases are found in plants, animals, and microorganisms.

OXIDOREDUCTASE-Oxidoreductases are another group of enzymes that catalyze the oxidation/reduction reaction. Oxidoreductases play a crucial role in foods in terms of taste, texture, shelf life, appearance, and nutritional value. Lipoxygenase, Lactoperoxidase, Polyphenol oxidase, peroxidase, Horseradish peroxidase, Lactoperoxidase, Catalase etc are commonly used in industries

\section{APPLICATIONS OF IMMOBILIZED ENZYME IN FOOD SECTOR}

Microbial enzymes have been used in the food industry for centuries. They also had applications in the leather industry, such as using dung for preparation of hides (Underkofler et al., 1958). In the 1930s, enzyme technology was used for the first time in the food industry, to clarify fruit juice.

\subsection{Dairy Industry}

Lipases are commonly used in the dairy industry to hydrolyze milk fat, and current applications of lipases in the dairy industry include cheese ripening, flavor enhancement, manufacturing cheese-like products, and lipolysis of cream and butterfat . Cheese texture is dependent on fat content so lipases that release short-chain fatty acids (C4 and C6) develop the sharp and tangy flavor, whereas release of medium-chain fatty acids (C12 and C14) causes a soapy taste in the product (Hasan et al., 2006). Lipases are also used for enzyme-modified cheeses (EMC) to liberate fatty acids at sn-1 and sn-3 positions on the glycerol backbone (Houde et al., 2004). EMC find applications in the food industry to add cheese flavor to salad dressings, dips, soups, sauces, and snacks.

Proteases have a broad application in the food industry. In the dairy industry, milk-coagulating enzymes (animal rennin, microbial coagulants, engineeredchymosin) are extensively used for cheese making. Chymosin has advantages over animal rennin due to its specific activity and availability. The protease-producing GRAS microorganisms are Mucormichei, Bacillus subtilis, and Endothiaparasitica.

Oxidoreductases are employed in the pasteurization of eggs and cheese by $\mathrm{H} 2 \mathrm{O} 2$, desugaring of eggs prior to spray drying, preservation of raw milk, and elimination of cooked flavor of UHT (ultra high temperature) pasteurized milk.

The most commonly used lactases for immobilization are obtained from E. coli and A. niger. The lactase enzyme immobilized on Teflon stirring bars that are coated with a polymer polyisocyanate was stable up to $\mathrm{pH} 8.75$. It can be used continuously for $137.6 \mathrm{~h}$ without appreciable losses in activity. d-Tagatose is a monosaccharide naturally present in dairy products, but in small amounts. Its sweetness is comparable with sucrose at $92 \%$ but has only $38 \%$ of the calories. From galactose, it can be produced via isomerization using the l-arabinose isomerase enzyme in an immobilized form obtained from Thermotoganeapolitana

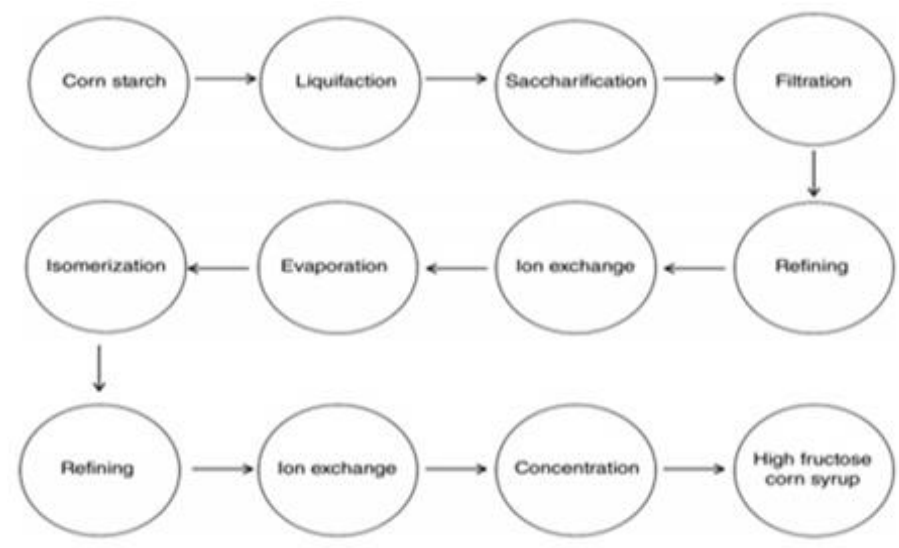

Fig2. Schematic for high-fructose corn syrup production from corn starch using immobilized glucose isomerize 


\subsection{Sugar Industry}

High-fructose corn syrup (HFCS) is used as a sweetener in beverages and foods. It has fewer calories and is cheaper than sucrose. In the United States, corn is the main raw material used to produce glucose syrup by hydrolysis of starch in the presence of amylases. After glucose syrups are produced, glucose High-fructose corn syrup (HFCS) is used as a sweetener in beverages and foods. It has fewer calories and is cheaper than sucrose. In the United States, corn is the main raw material used 6-7days of fermentation time with large-scale fermentation and a large storage capacity. The immobilization technique is used in the brewing industry by the entrapment of yeast cells to increase their concentration; this reduces the process time. Immobilized yeast cells reduce processing time without affecting the product quality.

Most alcoholic beverages, such as whiskey, vodka, and brandy, are produced from sugar containing raw materials. Malted barley, corn, milo, and rye are common raw materials for alcohol fermentation in the United States (Bigelis, 1993). The raw material is cooked to gelatinize the starch for enzymatic degradation, and cooled to room temperature before saccharification by amylase is performed. Fungal amylase, from Aspergillus or Rhizopus spp., is used for saccharification, because it increases the reaction rate while a complete saccharification is performed. It also produces fewer by-products, for example, maltose, isomaltose, and oligosaccharides, that are not fermentable by yeast.

\subsection{Cocoa Industry}

Cocoa butter fat is a high-value product with desired properties such as melting point, snap, and gloss. It also provides a cooling sensation and smoothness for chocolate (Hasan et al., 2006; Houde et al., 2004). However, a cheaper substitute for cocoa butter, palm oil, has a melting point of $23 \mathrm{C}$, is liquid at room temperature, and is a low-value product. Lipases are used to convert palm oil into cocoa butter substitute by transesterification reactions. Such modification of less expensive fats, such as shea butter, salt fat, and palm oil, can be obtained by transesterification and provides cheap substitutes for cocoa butter.

\subsection{Juice Industry}

In juice and wine making, Aspergillusnigerderivedpectic enzymes are commonly used. Sparkling clear juices, cloudy juices, and unicellular products are created by pectic enzyme applications in the juice industry. Enzymes for sparkling juices are employed to increase the yield and clarification of juice (Grassin\&Coutel, 2010; Kashyap et al., 2001). Filtration time is reduced up to 50\% when fruit juices are processed with pectic enzymes. Clarification is affected by $\mathrm{pH}$, temperature, enzyme concentration, and enzyme contact time. Lower $\mathrm{pH}$ will induce clarification rather than high $\mathrm{pH}$, while elevated temperature will also increase the clarification rate as long as they enzyme is not denatured (Kilara, 1982). Apple, pear, strawberry, raspberry, blackberry, and grape juice are some examples of sparkling clear juices. Another use of pectic enzymes in juice industry is stabilizing the cloud of citrus juices, purees, and nectars. Orange, lemon, mango, apricot, guava, papaya, pineapple, and banana are processed with enzymes to maintain a cloudy texture.

\section{CONCLUSION}

In the past few years, several studies have been done with a primary focus on the development of immobilized enzymes for future commercial use. Though the immobilized enzyme has several advantages in food processing, there are very few successful examples of immobilized enzymes in food processing. The immobilized glucose isomerase is used in the production of HFCS. The immobilized lipases are used in the production of diacylglycerols and transfree fats and/or oils. The main drawback of the immobilized enzyme system is its economics, which offset most of the other benefits of immobilized enzymes. Various other enzymes and their applications are at different stages of development. As well as the applications discussed, it has been suggested that enzymes may find extensive application in the production of flavourants in bread, beer, wine and other fermented foods as well as production of synthetic foods. Of course, numerous novel concepts have been attempted or are being pursued, such as: descaling of fish; modification of wort; beverage clarification; production of hydrolysate-based beverages for infants, geriatrics and invalids; enzymic determination as an index of food quality; food analyses; and removal of antinutritive factors from foods. The future of such processes and applications will depend heavily on economics and regulatory decisions. While it might be true that the implementation of immobilized-enzyme systems to date has not lived up to initial, 
optimistic expectations, the outlook is bright given current industry trends combined with the rapid evolution of immobilized-enzyme technology.

\section{REFERENCES}

[1] Alfredo Cassano, Integrated Membrane Processes in the Food Industry, Integrated Membrane Systems and Processes, First Edition, John Wiley \& Sons, Ltd. (2016) 36-55

[2] Beltsios KG et al Membrane science and applications. In: Handbook of Porous Solids. Weinheim, Germany: Wiley-VCH, (2008) pp. 2281-2433.

[3] SunitaAdhikari (Nee Pramanik), Application of Immobilized Enzymes in the Food Industry, Enzymes in Food Biotechnology (2019) 711-721

[4] Willaert, R., Verachtert, H., Bremt, K., Delvaux, F.R., Derdelinckx, G., Bioflavouring of foods and beverages. In: Application of Cell Immobilisation Biotechnology. (2005) 355-372.

[5] John E. Smith, Biotechnolgy, fifth edition, Cambridge university press,(2009) 73-109.

[6] Ali Demirci, GultenIzmirlioglu, and DuyguErcan, Fermentation and Enzyme Technologies in Food Processing, Food Processing: Principles and Applications, Second Edition,John Wiley \& Sons, Ltd. Published (2014) 107-130.

[7] Ahmad Homaei, Enzyme Immobilization and its Application in the Food Industry, Advances in Food Biotechnology, First Edition John Wiley \& Sons, Ltd. Published 2016 by John Wiley \& Sons, Ltd.,(2016) 145-156.

Citation: Sakshi Busamwar, et.al, "Application of Enzyme Immobilization in the Food Industry", International Journal of Advanced Research in Chemical Science, 7(2), pp. 6-10. DOI: http://dx.doi.org/10.20431/2349-0403.0702002

Copyright: () 2020 Authors, This is an open-access article distributed under the terms of the Creative Commons Attribution License, which permits unrestricted use, distribution, and reproduction in any medium, provided the original author and source are credited. 